\title{
Demographic data of patients with $\beta$-thalassemia major recorded in the electronic system in the north of Iran, 2016
}

\author{
MEHRNOUSH KOSARYAN, HOSSEIN KARAMI*, HADI DARVISHI-KHEZRI, ROSSETA AKBARZADEH, AILY \\ ALIASGHARIAN and KHADIJEH BROMAND \\ Thalassemia Research Centre, Hemoglobinopathy Institute, Mazandaran University of Medical Sciences, Sari, \\ Iran
}

\begin{abstract}
Background: Major thalassemia prevention project in Iran began in 1997. Mazandaran Province in northern Iran took effective steps in preventing the birth of patients with thalassemia major in the country. We report on the demographic status of patients with thalassemia major registered in the electronic system of Mazandaran Province in Iran.

Methods: The web-based application of the Mazandaran Thalassemia Registry (THRegistry) was designed based on the Net Framework platform in VB.Net and the 2014Sql Server database. The information source included clinical records in 14 thalassemic wards of the Mazandaran province hospitals. Epidemiological data of patients and date of transfusion, blood group, educational status, employment, marital status and having children were recorded.

Results: The study population was 1,725 patients including $889(51.5 \%)$ women and $836(48.5 \%)$ men with a mean age of $30 \pm 9.6$ years. A total of 188 patients born have been identified after the launch of the country's disease prevention plan in 1997. Prenatal diagnosis (PND) services were not used in 99 cases, PND result was mistaken in 6 other cases and in the remaining 5 cases PND was performed. A total of 1,314 patients (76.2\%) were blood transfusion dependent thalassemia (TDT). Mean haemoglobin level in male and female patients was $9.4 \pm 6.3$ and $9.01 \pm 5.1 / 100 \mathrm{~g} / \mathrm{dl}$, respectively. The mean age of onset of transfusion was $4 \pm 6.3$ years. A total of 382 cases $(22.1 \%)$ of married patients had a child of their own.

Conclusions: The system is a good resource to design descriptive and cohort studies, survival studies, therapeutic planning, and observation of the success of the major thalassemia prevention project.
\end{abstract}

Keywords: $\beta$-Thalassemia major, demography, descriptive, electronic registration system, registries, Iran

\section{Introduction}

Haemoglobinopathies constitute the most prevalent monogenic disorders and present as systemic healing problems in $71 \%$ countries worldwide (Modell et al., 2001). Incidence of haemoglobinopathies are high in the Middle East, the Indian Sub-continent, Southeast Asia and the Pacific Islands (De Sanctis et al., 2017). $\beta$-thalassemia major is a severe anaemia, which is the most common hereditary disease in the world. About $1.5 \%$ of the global population are carriers of $\beta$-thalassemia. It is also an important public health disease in the Islamic Republic of Iran and the province of Mazandaran (Samavat \& Modell 2004; Kosaryan et al., 2017). The inheritance of the disease is autosomal recessive. Gene carriers are known as thalassemia minor patients who are considered to be healthy. Those with two defective genes that have received them from their minor parents will suffer from severe and progressive anaemia and are considered as major thalassemia patients. People with major thalassemia are divided into two groups: transfusiondependent $\beta$-thalassemia major (TDT) caused by severe anaemia and the non-transfusiondependent thalassemia (NTDT) - a form of the disease that does not usually require blood transfusion (Abolghasemi et al., 2007).

In the efforts to control hereditary diseases, the World Health Organization introduced measures to register the epidemiological data of haemoglobin disorders, including $\beta$-thalassemia major (Modell \& Darlison, 2008). Project for prevention of thalassemia patients in Iran began in 1997. Mazandaran province has been successful and effective in preventing birth of thalassemia major patients (Samavat \& Modell 2004; Khorasani et al., 2008).

\footnotetext{
*Correspondence E-mail: karami_oncologist@yahoo.com
} 
In the past, patients of thalassemia in Iran died of severe anaemia, and few who survived died in the early years of their youth. In the last three decades, due to proper care services, patients' lifespan has increased. However, the treatment management became more complex and required the intervention of specialists in various branches of medicine (Kosaryan et al., 2007). Currently, these patients receive health care services in 15 hospitals in northern Iran across the province and 14 health centres are under the supervision of Mazandaran University of Medical Science (MUMS) (Kosaryan et al., 2017). Systematic registries are designed to provide accurate, comprehensive and up-to-date information on various aspects of the disease management (Kosaryan et al., 2017a,b). This information is used for descriptive and cohort research, drug and treatment planning, and monitoring of prevention activities. The present article aims to report the demographic status of thalassemic patients registered in the electronic system in Mazandaran Province in northern Iran.

\section{Materials and Methods}

The Mazandaran Thalassemia Registry (THRegistry) is a web-based application designed based on the Net Framework platform in VB.Net and 2014Sql Server database at thr.mazums.ac.ir. The information sources included patients' clinical records and their tests results in thalassemic wards of 14 hospitals in northern Iran. In order to inform the patients, the posters were attached on the wall of all wards to introduce the registration system for thalassemic patients. One of the nurses in each ward was responsible for registering patients in the system. Informed consent forms were signed and kept by patients or parents. The diagnosis of $\beta$-thalassemia major was based on the result of complete blood count ( $C B C)$ test, electrophoresis and some of patients by DNA analysis as standards.

Epidemiological data of patients (date of birth, sex) along with date of blood transfusion, blood group, educational status, employments, marital status and having children were recorded. In case of the births occurred in 1997 and later, three other options were recorded and extracted, including the use of prenatal diagnostic services, diagnosis error and non-abortion through asking parents and referring to the clinical records of patients. Also, in case of death of patients, the date and cause of death were recorded. The number and expertise of physicians practicing in each ward was explored and documented.

The system has the ability to plot for all variables by location of the hospital and the entire province in different seasons and years. The number and percentage of frequency, mean and standard deviation are calculated by the system. Registry software has ability to do statistics by 2014 Sql Server. All the registered patients who had already paper files were registered after taking their informed consent or from their parents/guardians.

\section{Results}

The registration of patients began in early 2016 and was completed by the end of November 2016. A total of 1,725 patients were registered. The data of this study showed that $95 \%$ of patients were registered in the system. $889(51.5 \%)$ and $836(48.5 \%)$ were female and male, respectively. The distribution of the number of patients based on the place of birth and the hospital is shown in Figure 1. 


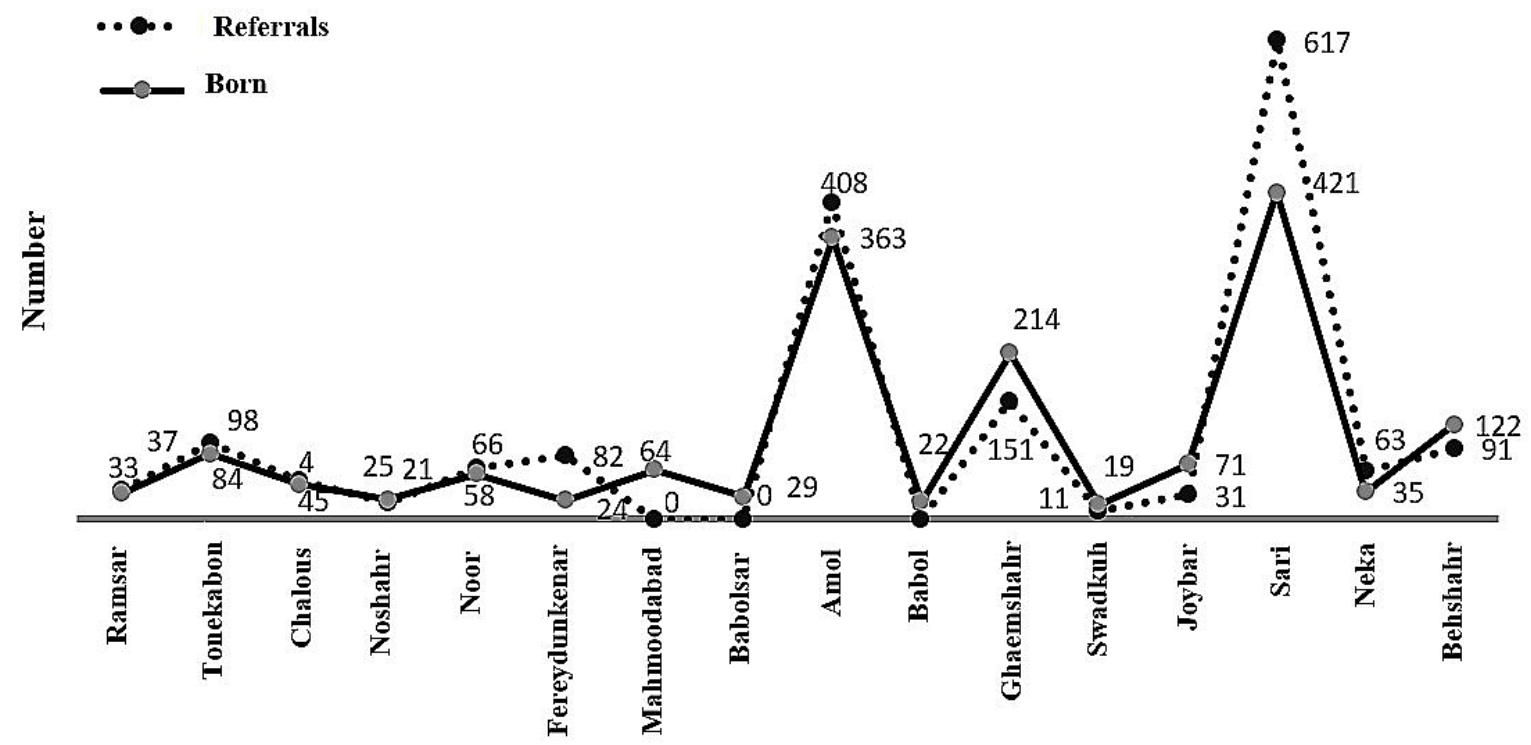

City

Figure 1: Distribution of patients with major thalassemia by city of birth and place of referral $(n=1,725)$

The mean age of patients was similar in men and women and was $30 \pm 9.6$ years (Figure 2). The distribution of patients by year of birth is shown in Figure 3. Most of the patients were aged between 25 and 40 years. In most of the age categories, the females accounted for the majority of the patients (Figure 3 ).

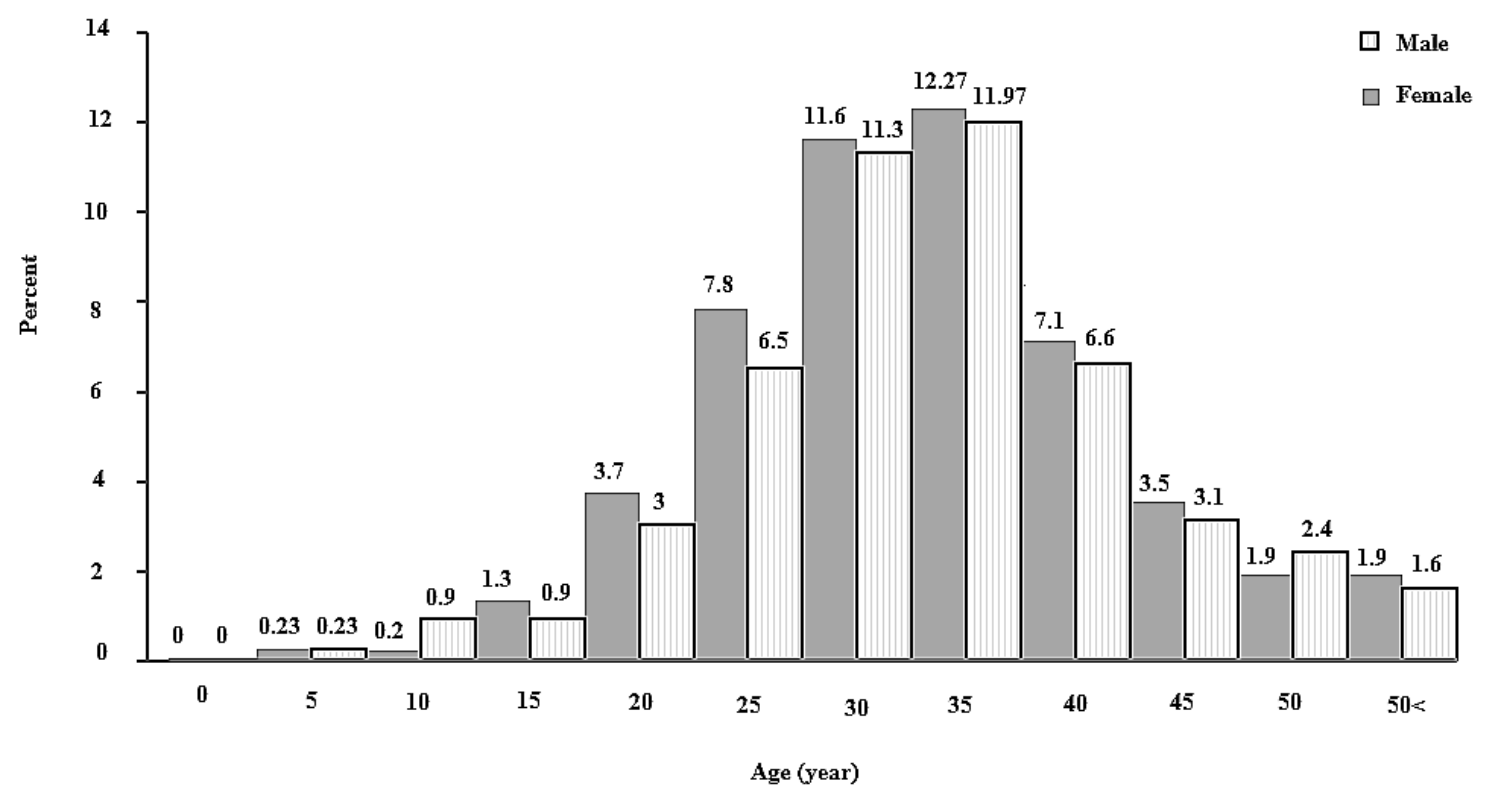

Figure 2: Distribution of patients with thalassemia major in Mazandaran Province by age group and $\operatorname{sex}(n=1,725)$

The number of patients aged < 20 years was 188 since the start of the prevention project (1997) in Iran (Figure 3). A number of challenges faced by the project have been documented. They included failure to use prenatal diagnosis (PND) services in 99, error in the PND results in 6 cases and, PND was performed but no abortion was made in 5 cases. In 78 cases, the cause of the failure of the prevention project was not clear. 


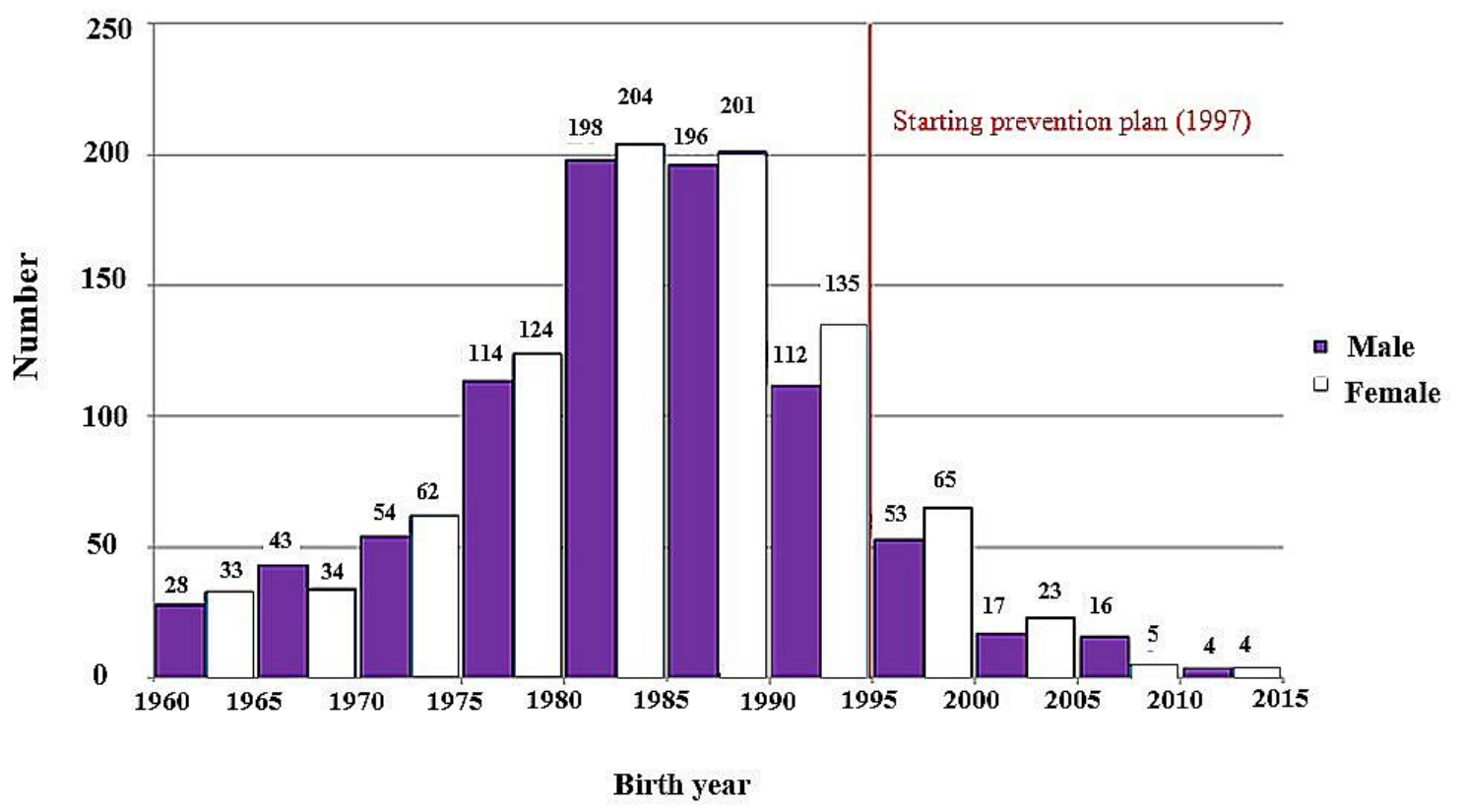

Figure 3: Distribution of patients with thalassemia major based on their year of birth

Since 2001 when the abortion strategy and insurance coverage for diagnosis and abortion was introduced, the number of patients was 69 people and an average of 4.3 people per year were recorded. A total of 1,314 patients (76.2\%) were blood transfusion-dependent thalassemia. Most of the patients belonged to Group O+ blood, followed by Groups A+ and B+ (Figure 4). None of the patient belonged to Group B-. In 8.6\% of patients, the blood group was not recorded.

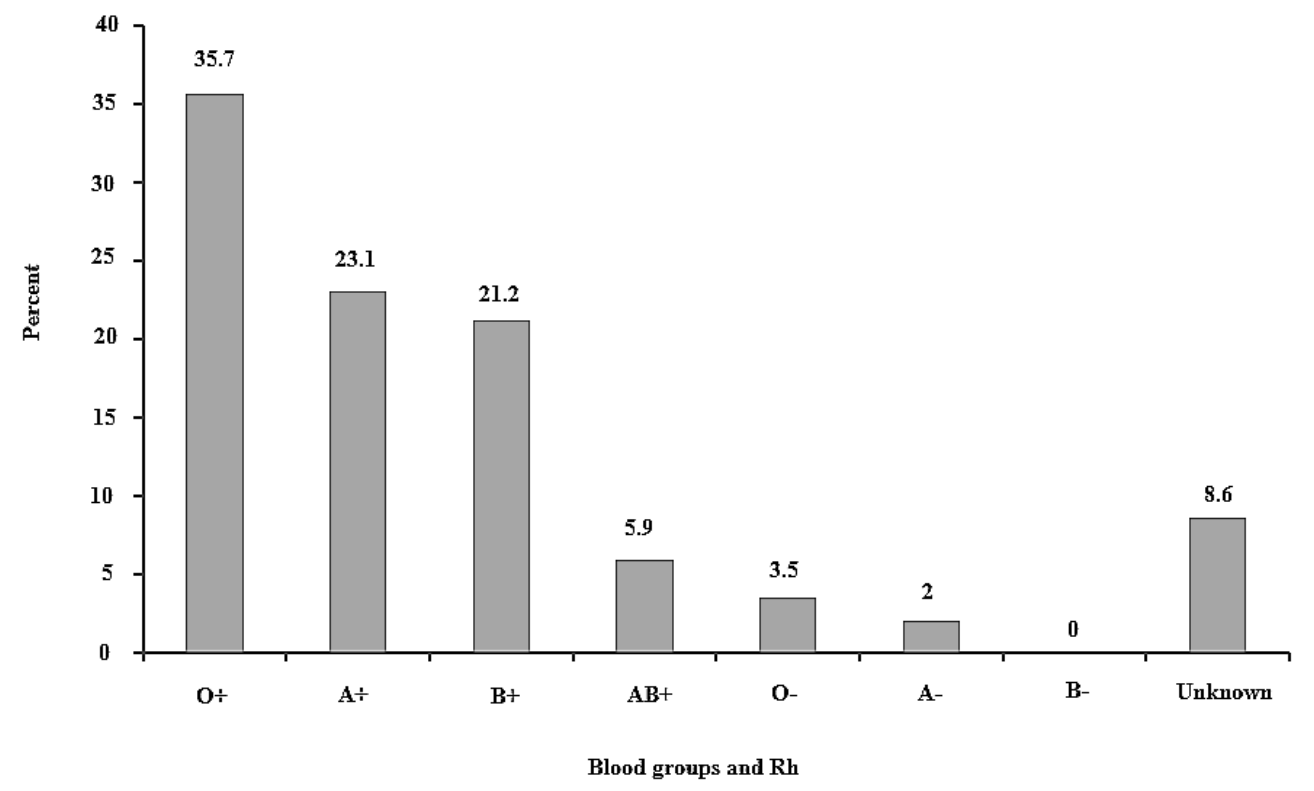

Figure 4: Distribution of patients with thalassemia major by blood group and $R h, 2016(n=1,623)$

The mean of haemoglobin in males and females was $9.4 \pm 6.3$ and $9.1 \pm 5.1 \mathrm{~g} / \mathrm{dl}$, respectively. The mean age of patients $(1,320)$ who were blood transfused was $4 \pm 6.3$ years old. About three quarters $(74.3 \%)$ of the patients started blood transfusions at the age of less than five years. Most patients began blood transfusion at the age of 2 years (38.7\%). About a quarter (24\%) of the cases had their first blood transfusion at the age of 1-year-old. Those who had blood transfusion at the age of 3 and 4 years accounted for $19.6 \%$ and $9.4 \%$ of the cases, respectively. Lower 
percent of patients began blood transfusions at the age of 5 years old (8.3\%). Also, the male to female ratio was $1 \pm 1.07: 1.1 \pm 1.19$.

The level of education was above the diploma in about one-third of patients. $28.2 \%$ of female and $23 \%$ of male among patients were singles. The percentage of married cases based on female and male were $16.2 \%$ and $19.4 \%$. Moreover, the distribution of engaged and divorced patients were $2.4 \%$ vs. $2.3 \%$ for female and $1.7 \%$ vs. $0.8 \%$ for male. $0.2 \%$ of female patients was widow. Marital status for $2.3 \%$ of female and $3.5 \%$ of male cases was unknown.

The results of this study showed that the mean age at menarche of the 175 girls examined was $15.4 \pm 1.6$ years which was considered to be a delayed puberty. The mean age at marriage was $21.8 \pm 4.9$ years. About half of the girls were treated for the onset and completion of puberty, and $21.9 \%$ of the women were pregnant using medical help. Most men raised no problems with fertility, except for 3 individuals whose wives underwent a microinjection technique.

A total of 382 cases $(22.1 \%)$ of married patients had at least a child. About a third (31.5\%; $\mathrm{n}=561$ ) of the patients were employed. In 2016 and up to the time of reporting (December 2016), 9 patients died. There were 3 women and 6 men. The mean age in males and females was $32.5 \pm$ 1.5 and $30 \pm 5.5$, respectively, and the cause of death was the complication of the disease and the accident in 8 cases.

\section{Discussion}

One of the many types of systematic registration is based on hospital records. The knowledge and experience of users is important in the proper recording of patients' information. The registry system of thalassemia major patients in northern Iran has used nurses working in the same wards to record patients' data. If physicians accurately record the necessary information in the records, the information will be easily transferred to the electronic form. Test result sheets are also a good source for finding information. In the case of incomplete data, patients or parents can be interviewed; however, it will lead to an in increase in costs, time and possibility of recall bias. Some registries are carried out by sending questionnaires to people who are informed or phone calls (Thuret et al., 2010). The choice of method depends on the facilities and culture of the community. The distribution chart of patients in different cities shows that the disease prevalence in the cities of Sari and Amol was higher than other cities; in other words, there are fewer patients in the two eastern and western parts of Mazandaran province (north of Iran) geographically.

The relationship between the place of birth and the place of treatment shows that patients basically refer to the nearest treatment site. In other words, the supply of healthcare services in all cities was more or less the same. However, in centres where there are subspecialists in blood diseases or other disciplines, the number of patients who refer for counselling visits is likely to be higher (this is not recorded in the system). Since 2015, the treatment system has tried to register a patient in one centre; however, patients are free to refer for counselling visits. The present study showed that the mean age of registered patients in northern Iran is 30 years. In a previous study in Iran, Abolqasemi et al. (2007) reported a mean age of 15 years old and only $23 \%$ of patients were over the age of 20 . Our previous study in 2010 investigated the demographic status of patients referred to the thalassemic department in Sari and half of them were over the age of 20 years, with an average age of $23 \pm 8$ years (Karami et al., 2010). In 2016, $96 \%$ of patients were over 20 years old. This indicates a fairly good care provided for patients in Sari as northern city in Iran.

One of the medical indicators for patients is the level of haemoglobin in patients. It is recommended that haemoglobin levels be maintained above $10 \mathrm{~g} / \mathrm{dl}$ in order to prevent bone marrow over-activity and its complications (Azarkeivan et al., 2006). The present study showed that there is no age difference between male and female patients. It may be concluded that there is no sex preference among family while protecting their female and male patients. Hassanzadeh 
et al. (2017), suggested that the delay in initiating treatment for patients with thalassemia major was observed in $65 \%$ of patients; this delay was longer among girls, and was attributed to a form of sex choice. In our study, women-men ratio was equal in all age groups. In our study, patients' age at the first blood transfusion was $4 \pm 6.2$ years old which was an older age than that reported among thalassemia patients in the southern regions of Iran (Hassanzadeh et al., 2017). This difference is likely to be due to the severity of the anaemia and the type of beta globin gene mutation, or that the parents of the patients are more sensitive to the symptoms of anaemia. Souteh et al. (2008) have reported the IVSII-I ( $(\mathrm{P}) \mathrm{A}$ ) mutation as the common beta-globin gene mutation in Mazandaran province. Although this mutation is of the $\beta 0$ type, other factors increase the relative proportion of haemoglobin in patients to the extent that the findings reported that three quarters of the patients were in need of frequent transfusion (transfusiondependent thalassemia major).

Thalassemia prevention activities began in northern Iran in 1991 (Khorasani et al., 2008), and continued more strong at the national level in 1997 (Samavat \& Modell 2004; Abolghasemi et al., 2007). The significant success of this project was observed only after the adoption of the abortion strategy for the affected foetuses and the coverage of costs by the insurance organizations since 2001. The causes of failure in prevention included non-use of foetal diagnosis services, foetal diagnosis errors and lack of abortion despite a proper diagnosis. There were 78 unspecified cases, with a few number of this group observed in children born using laboratory fertility services and adopted children. It was not possible to register these cases in the registration system because of the ethical sensitivity and total confidentiality of the subjects.

The present research showed that about $40 \%$ of patients are married and about two thirds of them have children. In the past, due to the changing face of patients and the numerous complications of thalassemia, marriage and childbirth were not possible for most patients. Currently, patients are able to play an active role in society, have jobs and form a family. However, some patients need help with fertility.

\section{Competing interests}

The authors declare that there is no conflict of interest

\section{Acknowledgments}

Thereby, the authors would like to appreciate the respected vice chancellor of research and technology of Mazandaran University of Medical Sciences for their approval and comprehensive support of the project. The vice chancellor of treatment of the University is appreciated for the full support of the establishment of the system. Vice chancellor for development and coordination of research, the Ministry of Health and Medical Education is also appreciated for supporting and allocating funds and promoting the project at the national level. Also, we express our thanks and gratitude to all patients and their parents who trust us and provide us with this valuable information.

\section{Authors' contribution}

MK: Conceived the idea and designed the study, literature search and reviewed the manuscript for intellectual content. HK: assisted designing the study and reviewed the manuscript for its intellectual content. HDKH: acquired the data, assisted literature search and the manuscript drafting. RA: reviewed the manuscript for its intellectual content. AA and KHB: acquired the data. 


\section{References}

Abolghasemi, H., Amid, A., Zeinali, S., Radfar, M.H., Eshghi, P., Rahiminejad, M.S., Ehsani, M.A., Najmabadi, H., Akbari, M.T. \& Afrasiabi, A. (2007) Thalassemia in Iran: epidemiology, prevention and management. Journal of Pediatric Hematology/Oncology 29: 233-238.

Azarkeivan, A., Eshghi, P., Karimi, M., Badiei, Z. \& Aghighi, M. (2006) Treatment Guide Book in Thalassemia Patients. Ministry of Health and Medical Education, Tehran, Iran.

De Sanctis, V., Kattamis, C., Canatan, D., Soliman, A.T., Elsedfy, H., Karimi, M., Daar, S., Wali, Y., Yassin, M., Soliman, N., Sobti, P., Al Jaouni, S., El Kholy, M., Fiscina, B. \& Angastiniotis, M. (2017) $\beta$-Thalassemia Distribution in the Old World: an Ancient Disease Seen from a Historical Standpoint. Mediterranean Journal of Hematology and Infectious Diseases 9(1): e2017018.

Hassanzadeh, J., Mirahmadizadeh, A., Karimi, M. \& Rezaeian, S. (2017) Delay in diagnosis of hemoglobulinopathies (thalassemia, sickle cell anemia): a need for management of thalassemia programs. Iranian Journal of Pediatrics 27(2).

Karami, H., Kowsaryan, M., Vahidshahi, K., Karami, H., Shahmohammadi, S., Mahdavi, M., Hashemi, M.B., Abdolmaleki, E.Y., Majdi, M. \& Ahangar, M. (2010) Assessment of demographic, clinical and laboratory status of patients with thalassemia major and intermedia referred to thalassemia research center in Sari, Iran, during 2007-2009. Pajoohandeh Journal 15: 186-192.

Khorasani, G., Kosaryan, M., Vahidshahi, K., Shakeri, S. \& Nasehi, M.M. (2008) Results of the national program for prevention of $\beta$-thalassemia major in the Iranian Province of Mazandaran. Hemoglobin 32: 263-271.

Kosaryan, M., Karami, H. \& Akbarzadeh, R. (2017a) Designing an electronic registry for patients with beta thalassemia major for Mazandaran Province, IR Iran 2016. International Journal of Caring Sciences 10: 575.

Kosaryan, M., Karami, H., Alipour, A., Akbarzadeh, R., Aliasgharian, A., Masoudinejhad, M., Darvishi-Khezri, H. \& Sari, I. (2017b) First report of the electronic registry of patients with beta thalassemia major in Mazandaran Province, Iran. Journal of Mazandaran University of Medical Sciences 27: 54-65.

Kosaryan, M., Vahidshahi, K., Karami, H., Forootan, M.A. \& Ahangari, M. (2007) Survival of thalassemic patients referred to the Boo Ali Sina teaching hospital, Sari, Iran. Hemoglobin 31: 453-462.

Modell, B. \& Darlison, M. (2008) Global epidemiology of haemoglobin disorders and derived service indicators. Bulletin of the World Health Organization 86: 480-487.

Modell, B., Khan, M., Darlison, M., King, A., Layton, M., Old, J., Petrou, M. \& Varnavides, L. (2001) A national register for surveillance of inherited disorders: beta thalassaemia in the United Kingdom. Bulletin of the World Health Organization 79: 1006-1013.

Samavat, A. \& Modell, B. (2004) Iranian national thalassaemia screening programme. BMJ 329(7475): 1134-1137.

Thuret, I., Pondarré, C., Loundou, A., Steschenko, D., Girot, R., Bachir, D., Rose, C., Barlogis, V., Donadieu, J. \& de Montalembert, M. (2010) Complications and treatment of patients with $\beta$-thalassemia in France: results of the National Registry. Haematologica 95: 724729.

Zafari $M$ and Kosaryan M (2014). "Marriage and child bearing in patients with transfusion-dependent thalassemia major." Journal of Obstetrics and Gynaecology Research 40(8): 1978-1982. 